\title{
Abnormal Cell Properties and Down-Regulated FAK-Src Complex Signaling in B Lymphoblasts of Autistic Subjects
}

\author{
Hongen Wei, ${ }^{, \dagger}$ Mazhar Malik, ${ }^{*}$ Ashfaq M. Sheikh, ${ }^{*}$ \\ George Merz, ${ }^{\ddagger}$ W. Ted Brown, ${ }^{*}$ and Xiaohong Li* \\ From the Department of Neurochemistry* and the Digital \\ Microscopy Laboratory, ${ }^{\ddagger}$ New York State Institute for Basic \\ Research in Developmental Disabilities, New York, New York; \\ and the Department of Anatomy, ${ }^{\dagger}$ Second Military Medical \\ University, Shanghai, China
}

Recent studies suggest that one of the major pathways to the pathogenesis of autism is reduced cell migration. Focal adhesion kinase (FAK) has an important role in neural migration, dendritic morphological characteristics, axonal branching, and synapse formation. The FAK-Src complex, activated by upstream reelin and integrin $\beta 1$, can initiate a cascade of phosphorylation events to trigger multiple intracellular pathways, including mitogen-activated protein kinaseextracellular signal-regulated kinase and phosphatidylinositol 3-kinase-Akt signaling. In this study, by using $B$ lymphoblasts as a model, we tested whether integrin $\beta 1$ and FAK-Src signaling are abnormally regulated in autism and whether abnormal FAK-Src signaling leads to defects in B-lymphoblast adhesion, migration, proliferation, and IgG production. To our knowledge, for the first time, we show that protein expression levels of both integrin $\beta 1$ and FAK are significantly decreased in autistic lymphoblasts and that Src protein expression and the phosphorylation of an active site (Y416) are also significantly decreased. We also found that lymphoblasts from autistic subjects exhibit significantly decreased migration, increased adhesion properties, and an impaired capacity for IgG production. The overexpression of FAK in autistic lymphoblasts countered the adhesion and migration defects. In addition, we demonstrate that FAK mediates its effect through the activation of Src, phosphatidylinositol 3-kinase-Akt, and mitogen-activated protein kinase signaling cascades and that paxillin is also likely involved in the regulation of adhesion and migration in autistic lymphoblasts. (Am J Patbol 2011, 179:66-74; DOI: 10.1016/j.ajpath.2011.03.034)
Autism is a severe neurodevelopmental disorder characterized by problems in communication, social skills, and repetitive behavior. Susceptibility to autism is clearly attributable to the interplay of genetic and environmental factors, ${ }^{1-10}$ but the etiology of this disorder is unknown and no biomarkers have yet been proved characteristic of autism. Recent research ${ }^{11}$ is beginning to depict three major pathways as being potentially involved in the pathogenesis of autism (ie, reduced cell migration, excitatory-inhibitory imbalance, and abnormal synaptogenesis). The reelin protein encoded by the RELN gene plays a pivotal role in neuronal cell migration and the prenatal development of neural connections. ${ }^{12}$ Reelin has coexpressed with HAR1F, which is a novel RNA gene expressed specifically in Cajal-Retzius neurons in the developing human neocortex from gestational week 7 to 19 (a crucial period for cortical neuron specification and migration). ${ }^{13}$ Linkage between $R E L N$ and autism is a replicated genetic finding in autism research, and reelin has been reduced in the serum and brain specimens of autistic subjects. ${ }^{14,15}$ Reelin can act through $\alpha 3 \beta 1$ integrins and exert proteolytic activity on extracellular matrix (ECM) proteins, which are crucial for neuronal migration. ${ }^{16}$ Most recently, emerging evidence ${ }^{17}$ indicates that synaptic cell adhesion pathways are disrupted in some

Supported by the NYS Office for People with Developmental Disabilities, the Rural India Charitable Trust. Resources were provided by the Autism Genetic Resource Exchange (AGRE) Consortium and the participating AGRE families. AGRE is a program of Autism Speaks and is supported, in part, by grant 1U24MH081810 from the National Institute of Mental Health to Clara M. Lajonchere (principle investigator), and Autism Genetic Resource Exchange (AGRE) Consortium and the participating AGRE families.

Accepted for publication March 21, 2011.

CME Disclosure: The authors did not disclose any relevant financial relationships.

Current address of H.W., Department of Anatomy, Second Military Medical University, Shanghai, China.

Address reprint request to Xiaohong Li, M.D., Ph.D., Department of Neurochemistry, New York State Institute for Basic Research in Developmental Disabilities, 1050 Forest Hill Rd, Staten Island, New York, NY 10314. E-mail: xiaohong.li@opwdd.ny.gov or xiaohongli99@gmail.com. 


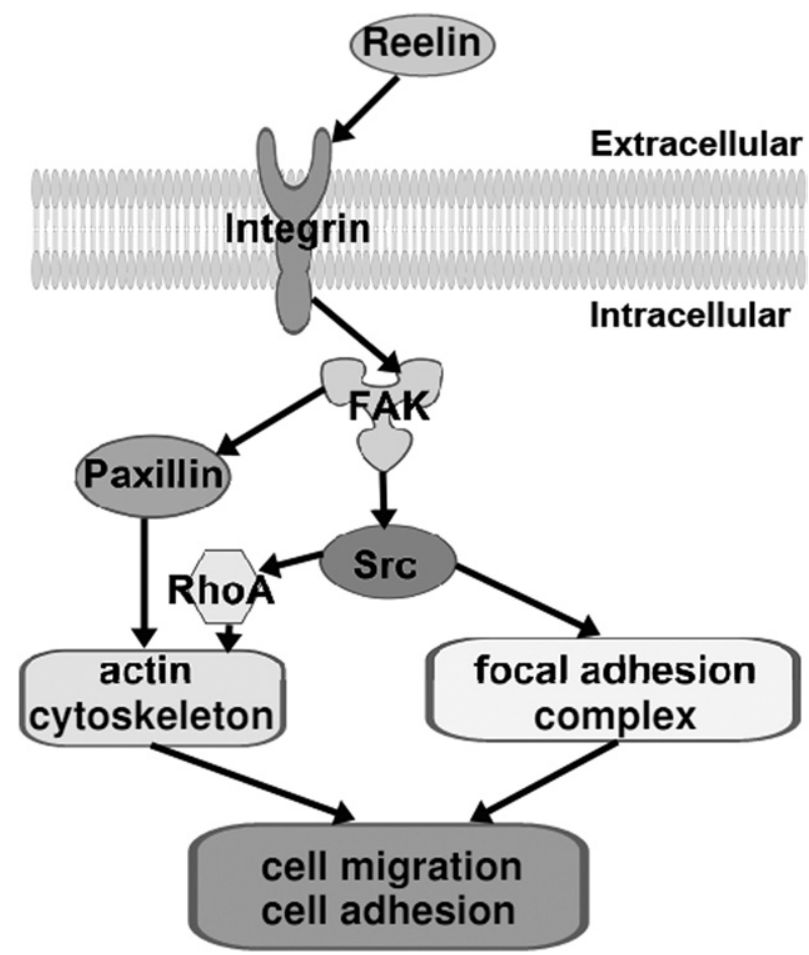

Figure 1. Signaling pathways of FAK and Src, controlling actin cytoskeletal rearrangement, and FAs. Integrin $\beta 1$ can be activated by reelin and induces recruitment and stimulation of FAK and Src, which control cell migration and adhesion via regulation of the actin cytoskeleton and FA complex. Activated FAK can also recruit paxillin in the regulation of cell migration and adhesion. RhoA indicates Ras homolog gene family, member A.

subjects with autism. However, to our knowledge, no studies have examined whether cell adhesion and migration are abnormally presented in autism and whether the focal adhesion kinase (FAK)-Src signaling pathway, which is essentially involved in the regulation of cell migration and adhesion, is abnormally regulated in autism.

FAs are sites of cell attachment to the ECM, where transmembrane integrins link the ECM to the cytoskeleton. ${ }^{18}$ Among the proteins localized to the FAs are FAK, Src family kinases, p21-activated kinase, the scaffolding protein paxillin, and the actin-binding proteins vinculin and talin $^{19}$ (Figure 1).

FAK was originally identified as a nonreceptor protein tyrosine kinase and may play a crucial role in cell adhesion and migration. ${ }^{20}$ As shown in Figure 1, FAK is activated by receiving signaling from the upstream integrins; FAK undergoes autophosphorylation at Y397, which generates a binding site for $\mathrm{Src}$ via the $\mathrm{SH} 2$ domain and activates Src. ${ }^{21-23}$ The activated Src further phosphorylates FAK on other tyrosine residues (Y576/577 and Y925), leading to the full activation of FAK. ${ }^{22,23}$ The activated FAK-Src complex then initiates a cascade of phosphorylation events and new protein-protein interactions to trigger multiple intracellular signaling pathways. Many studies $^{24}$ have shown that FAK facilitates the generation of integrin-stimulated signals to downstream targets, such as the mitogen-activated protein kinase (MEK)-extracellular signal-regulated kinase (ERK) 2 and the c-Jun $\mathrm{N}$-terminal kinase (JNK)-MEK cascades. FAK can also activate the phosphatidylinositol 3-kinase (PI3K)-Akt pathway by binding the p85 subunit of PI3K through autophosphorylation of Y397. ${ }^{25}$ The activation of FAK leads to several processes, including cell adhesion, migration, chemotaxis, proliferation, and survival. ${ }^{26}$ In addition, recent studies ${ }^{27-30}$ have revealed important functional roles of FAK in cortical basement membrane assembly and/or remodeling, neural migration, dendritic morphological characteristics, axonal branching, and synapse formation.

Taken together, we hypothesize that the ECM proteins FAK-Src and their downstream signaling pathways are abnormally regulated in autism, which may lead to defects in cell adhesion, migration, proliferation, and cell functioning. In this study, we used B lymphoblasts from autistic subjects and age-matched controls to test this hypothesis. Lymphoblast cell lines may be a valuable tool for identifying genes associated with autism and provide an alternative approach for understanding the biological features and genetics of autism. ${ }^{31}$ For the first time, to our knowledge, we show that the protein expression levels of both integrin $\beta 1$ and FAK are significantly decreased in autistic lymphoblasts. Src protein expression is also significantly decreased; and the Y416 active site is hypophosphorylated, whereas phosphorylation on the Y527 inhibitory site remains unchanged. These results suggest that FAK-Src signaling activity is reduced in autistic lymphoblasts. We also found that lymphoblasts from autistic subjects exhibit significantly decreased migration and have increased adhesion properties and impaired IgG production. Overexpression of FAK in autistic lymphoblasts can counter the adhesion and migration defects. In addition, we demonstrate that FAK mediates its effect through the activation of Src, PI3K-Akt, and MEK signaling cascades. Furthermore, we demonstrate that paxillin, which is a scaffolding molecule mediated by the FAK-Src complex, may also be involved in the regulation of autistic lymphoblast migration because paxillin protein expression is decreased in autistic lymphoblasts.

\section{Materials and Methods}

\section{Study Materials}

All antibodies used in the study were purchased from Cell Signaling Technology (Danvers, MA). His-FAK fusion protein, inhibitors of PI3K (wortmannin), Akt, and Src (SU6656) were purchased from Sigma-Aldrich (St. Louis, MO). The MEK inhibitor (PD98059) was purchased from Cayman Chemical (Ann Arbor, MI).

\section{Study Subjects}

The lymphoblast cell lines of eight autistic patients and eight healthy siblings were obtained from the Autism Genetic Resource Exchange (AGRE). Donors with autism fit the diagnostic criteria of the Diagnostic and Statistical Manual of Mental Disorders-IV, as confirmed by the Autism Diagnostic Interview-Revised. Participants were excluded from the study if they were diagnosed as having fragile $X$ syndrome, epileptic seizures, obsessive-com- 
pulsive disorder, affective disorders, or any additional psychiatric or neurological diagnoses. From the information provided in the AGRE database, the age of the eight autistic subjects used in our study is $7.3 \pm 2.3$ years and the age of the siblings is $11.3 \pm 1.9$ years (mean \pm SEM) (the ages of three siblings are not available). The difference between the two groups based on the available information is significant $(P=0.046)$. There is no medication listed for siblings. For the autistic subjects, four have no record of medication history, whereas two take Prozac (fluoxetine) for antidepression, one takes carbamazepine for antiseizure, and one takes a carnitine supplemental nutrient.

\section{Lymphoblast Culture}

Lymphoblasts obtained from AGRE were cultured in RPMI 1640 medium, supplemented with $1 \%$ penicillinstreptomycin and $10 \%$ fetal calf serum (culture materials from Invitrogen, Carlsbad, CA). The cells were fed with fresh medium every 3 to 4 days. The B-lymphoblast cell lines from AGRE were derived from peripheral blood lymphocytes, immortalized with Epstein-Barr virus, and treated with cyclosporin A. Lymphoblast cultures are supplied in T25 tissue culture flasks that have been filled to capacity with carbon dioxide-equilibrated medium to provide sufficient nutrients for extended transport times. Cells from autistic patients and control subjects were matched for passage number, which are one to two passages from the time of transformation. All samples in the present study were cultured and harvested at the same time ( 3 days after passaging) using the same medium preparation and protein isolation reagents.

\section{Western Blot Analysis}

Western blot analysis was performed as previously reported. ${ }^{32}$ Antibodies to integrin, FAK, Src, phosphorylated Src, and paxillin were purchased from Cell Signaling and used at concentrations suggested by the supplier.

\section{Cell Adhesion Assay}

Control lymphoblasts, autistic lymphoblasts, and autistic lymphoblasts transfected with His-FAK were used in this study. A total of 5000 cells were plated per well in 96-well tissue culture plates precoated with recombinant human intercellular adhesion molecule 1 (R\&D Systems, Minneapolis, MN) at a concentration of $10 \mathrm{mg} / \mathrm{mL}$. After 6 hours of attachment, unattached cells were removed by aspiration and adherent cells were quantitated by the colorimetric aqueous 3-(4,5-dimethylthiazol-2-yl)-5-(3-carboxymethoxyphenyl)-2-(4-sulfophenyl)-2H-tetrazolium (MTS) assay (CellTiter $96 \mathrm{AQ}_{\text {ueous }}$ One Solution kit; Promega, Fitchburg, WI).

\section{Modified Boyden Chamber Migration Assays}

Control lymphoblasts, autistic lymphoblasts, and autistic lymphoblasts transfected with His-FAK were labeled with fluorescent calcein-AM (BD Biosciences, Bedford, MA). A total of 10,000 labeled cells treated with or without 0.1 $\mu \mathrm{mol} / \mathrm{L}$ wortmannin, $5 \mu \mathrm{mol} / \mathrm{L}$ Akt inhibitor, $5 \mu \mathrm{mol} / \mathrm{L}$ SU6656, and $50 \mu \mathrm{mol} / \mathrm{L}$ PD98059 were plated in $0.8 \mathrm{~mL}$ RPMI 1640 medium plus 1\% fetal bovine serum in each well of 24-well chambers adapted for the FluoroBlok (BD Biosciences) apparatus. After 4 hours, 5\% fetal bovine serum was added to the lower chamber medium to establish a $1 \%$ to $5 \%$ serum gradient and migration of cells from the upper to lower chamber, quantitated at 14 hours using a microplate reader (CytoFluor 4000; MTX Lab Systems, Vienna, VA).

\section{Cell Proliferation Assay}

A total of 20,000 control and autistic lymphoblasts were plated per well in 96-well tissue culture plates. After 48 hours' incubation at $37^{\circ} \mathrm{C}$, cell proliferation was determined by the colorimetric aqueous MTS assay (CellTiter $96 \mathrm{AQ}_{\text {ueous }}$ One Solution kit). The plate was read on a $V_{\max }$ kinetic microplate reader (Molecular Devices, Sunnyvale, CA) at $490 \mathrm{~nm}$.

\section{Measurement of Ig Secretion}

For the investigation of Ig formation, B-lymphoblast cell lines were cultured at a cell density of $5 \times 10^{5} / \mathrm{mL}$ in 200 $\mu \mathrm{L}$ of culture medium, consisting of RPMI 1640 medium containing $10 \%$ fetal calf serum in 96-well plates for 3 days. Supernatants of autistic and control cells were harvested, centrifuged at $12,000 \times g$ for 10 minutes in a microcentrifuge, and stored at $-20^{\circ} \mathrm{C}$. Ig concentrations were measured by a human IgG enzyme-linked immunosorbent assay kit (Immunology Consultants Laboratory, Newberg, OR).

\section{Fluorescence Staining of Lymphoblasts}

Acridine orange plus propidium iodide double staining was used to determine the morphological characteristics of the lymphoblast cells. Cells from six autistic subjects and six controls were harvested and suspended in PBS solution. Cell suspension, $100 \mu \mathrm{L}$, was mixed with $2 \mu \mathrm{L}$ of a $1: 1$ mixture of propidium iodide $(1 \mathrm{mg} / \mathrm{mL}$; SigmaAldrich) and acridine orange ( $3 \mathrm{mg} / \mathrm{mL}$; Sigma-Aldrich). After a 5-minute incubation, $10 \mu \mathrm{L}$ of cell suspension was mounted onto a glass slide and examined at $\times 400$ magnification with a fluorescence microscope (Nikon, Melville, NY). Only the viable cells identified by a bright green nucleus with an intact structure were counted and measured.

\section{Morphometric Analysis}

Computer-assisted morphometry was performed using an image analysis program (Image-Pro Plus 6.0; Media Cybernetics, Bethesda, MD). The parameters analyzed were mean nuclear area, mean cytoplasmic area, and mean nuclear/cytoplasmic ratio. Ninety lymphoblasts from six autistic subjects and six controls were examined in this study. 


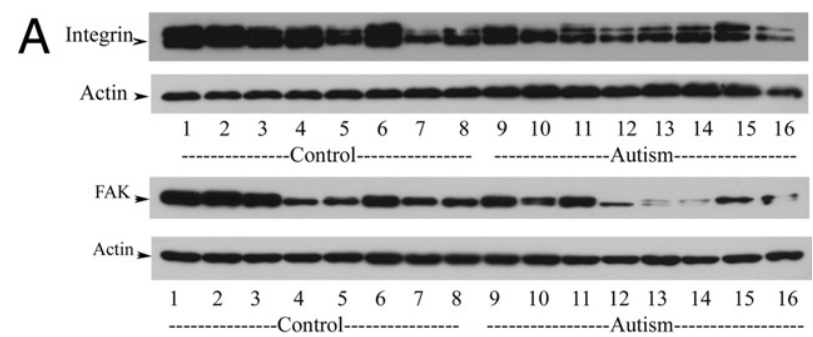

B
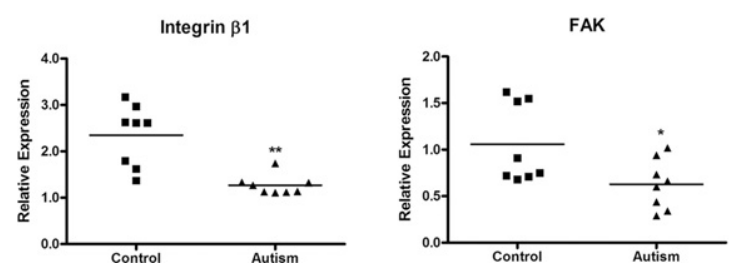

Figure 2. Integrin $\beta 1$ and FAK protein expression in autistic lymphoblasts. A: Western blot analyses of lymphoblast lysates using integrin $\beta 1$ antibody and FAK antibody. B: The blots shown in A were quantitated after normalization by actin. Data are shown as mean $\pm \operatorname{SE}(n=8)$. ${ }^{*} P<0.05,{ }^{* *} P<0.01$.

\section{Statistical Analysis}

We analyzed the statistical significance among groups with the unpaired t-test and analysis of variance with Fisher's protected least significant difference (PLSD) post hoc test using StatView 5.0 software (SAS Institute, Inc., Cary, NC). The samples shown in our study were all of the samples analyzed. All data are presented as the mean \pm SE. Significance was accepted at $P<0.05$.

\section{Results}

\section{Protein Expression Levels of Integrin $\beta 1$ and FAK Are Decreased in Autistic Lymphoblasts}

The total protein expression of FAK and its upstream activator integrin $\beta 1$ in both the autistic lymphoblasts and the controls were determined using Western blot assays. The bands representing the integrin $\beta 1$ (115- and 135$\mathrm{kDa}$ ) and FAK (125-kDa) proteins from both groups are shown in Figure 2A. Quantitative analysis indicates that the mean values of integrin $\beta 1$ and FAK expression are decreased by $46 \%(t=4.39, \mathrm{df}=14, P=0.0016)$ and $41 \%(t=2.42, \mathrm{df}=14, P=0.03)$, respectively, in the autistic lymphoblasts compared with the controls (Figure $2 \mathrm{~B}$ ).

\section{Protein Expression and Activity of Src Are Down-Regulated in Autistic Lymphoblasts}

Src is the protein FAK directly binds to and activates. ${ }^{22-24}$ The activated FAK-Src complex can initiate a cascade of phosphorylation events to trigger multiple intracellular signaling pathways. To further determine whether the decreased FAK activity affects Src, we examined total Src protein expression and Src phosphorylation. Src has two phosphorylation sites (Y416 and Y527). The phosphorylation of Y416 enhances Src function, whereas the phosphorylation of Y527 inhibits Src activities. ${ }^{33}$ Our re- sults showed that total Src protein expression in autistic lymphoblasts was decreased by $26 \%(t=8.329, \mathrm{df}=14$, $P<0.001$ ) (Figure 3). In addition, Src phosphorylation on the activating site (Y416) was reduced by a mean of $26 \%$ compared with controls $(t=2.620, \mathrm{df}=14, P=0.02)$ (Figure 3). We did not detect a significant change of Src phosphorylation on the inhibitory site (Y527) comparing the autistic with the control group $(t=0.1543$, df $=14$, $P=0.87$ ) (Figure 3). These results suggest that FAK-Src signaling activities were down-regulated in the autistic lymphoblasts.

\section{Autistic Lymphoblasts Exhibit Significant Defects in Migration, Adhesion, and the Function of IgG Production}

Next, we examined whether the compromised FAK-Src signaling has an effect on autistic lymphoblast properties, including migration, adhesion, and proliferation. Modified Boyden chamber migration assays were performed to examine cell migration. Our results showed that autistic lymphoblasts exhibited a $26 \%$ decrease in migration compared with the controls $(t=26.06, \mathrm{df}=8$, $P=0.0023$; Figure 4A). With a cell adhesion assay, we observed that the autistic lymphoblast adhesion to intercellular adhesion molecule 1 was increased by $15 \%(t=$ 2.91, df $=14, P=0.02$; Figure 4B). We did not detect a significant difference in cell proliferation between the autistic lymphoblasts and the controls $(t=0.95, \mathrm{df}=14$, $P=0.37$; Figure $4 C$ ) with a colorimetric aqueous MTS assay.

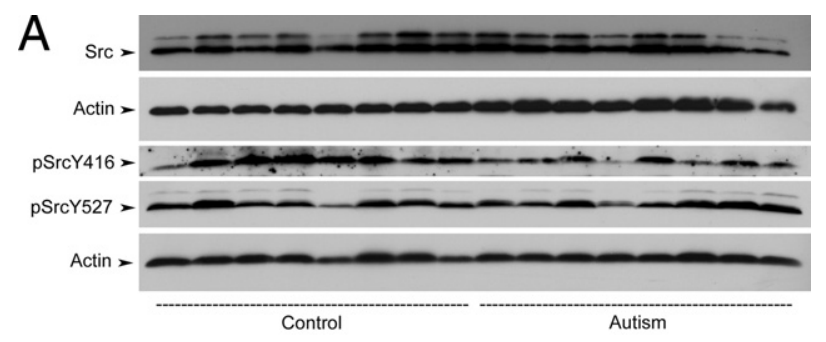

B

Src
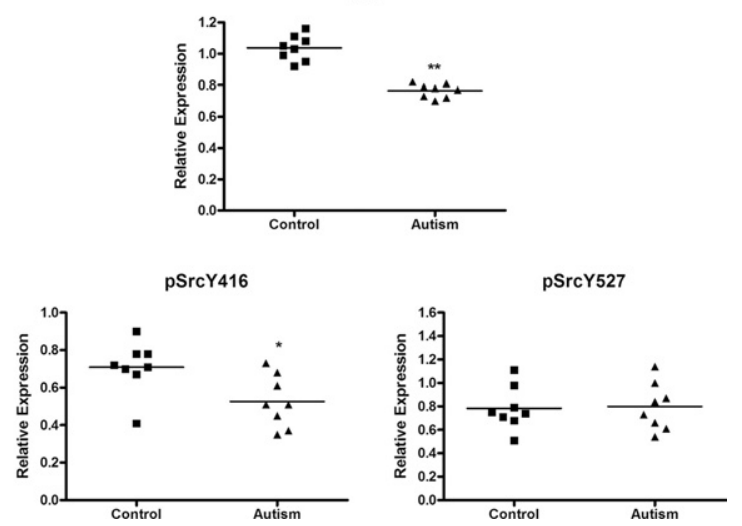

Figure 3. Src protein expression and phosphorylation in autistic lymphoblasts. A: Western blot analyses of lymphoblast lysates using Src antibody, phosphorylated Src (pSrc) (Y416) antibody, and pSrc (Y527) antibody. B: The blots shown in A were quantitated after normalization by actin. Data are shown as mean \pm SE $(n=8)$. ${ }^{*} P<0.05,{ }^{* * *} P<0.01$ 
A
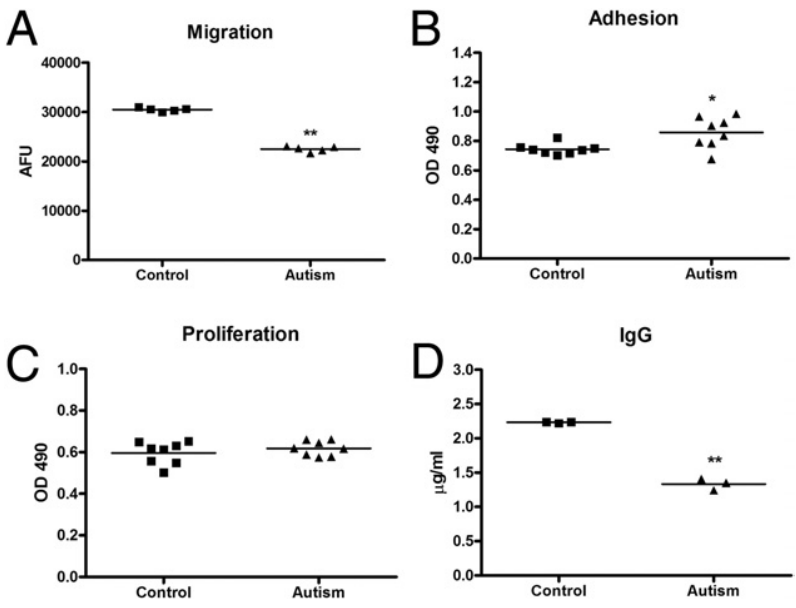

Figure 4. The profile of lymphoblast migration, adhesion, proliferation, and IgG production in autistic subjects. A: Modified Boyden chamber migration assays on lymphoblasts from autistic and control subjects. The number of migrated cells was determined by fluorescence reading at 14 hours. The values are mean \pm SE from five replicates. AFU indicates arbitrary fluorescence units. B: Comparison of 6-hour lymphoblast adhesion between the autistic and control groups. The values are mean $\pm \mathrm{SE}$ from eight replicates. C: Comparison of 48-hour lymphoblast proliferation between the autistic and control groups. The values are mean $\pm \mathrm{SE}$ from eight replicates. D: Comparison of IgG production between the autistic and control groups. The values are mean \pm SE from three replicates. ${ }^{*} P<$ $0.05,{ }^{* * *} P<0.01$

The formation and secretion of Igs are natural functions of B lymphoblasts. To evaluate whether B-lymphoblast function was impaired in the autistic cells because of altered adhesion and migration, we further assayed Ig production. B-lymphoblast cell lines from autistic and control subjects were cultured at a cell density of $5 \times$ $10^{5} / \mathrm{mL}$ using 2-mercaptoethanol (50 $\mu \mathrm{mol} / \mathrm{L}$ ) to induce cell differentiation. Ig concentrations were quantitated with a human IgG enzyme-linked immunosorbent assay kit. Our results showed that autistic B lymphoblasts produced significantly less Ig in response to 2-mercaptoethanol than controls $(t=17.92$, $\mathrm{df}=4, P<0.001$; Figure $4 \mathrm{D})$, indicating that B-lymphoblast function in autism was impaired.

\section{Autistic Lymphoblasts Exhibit No Significant Change in Morphological Characteristics}

To determine whether the changes in autistic lymphoblast properties are accompanied with changes in morphological characteristics, we examined three morphometric parameters, including mean nuclear area, mean cytoplasmic area, and mean nuclear/cytoplasmic ratio in autistic and control lymphoblasts with a computer-assisted morphometry program (Image-Pro Plus 6.0). Our results showed that there were no significant differences in mean nuclear area $(t=-0.38, \mathrm{df}=10, P=0.71)$, mean cytoplasmic area $(t=-0.63, \mathrm{df}=10, P=0.54)$, and nuclear/cytoplasmic ratio $(t=0.80, \mathrm{df}=10, P=$ 0.44 ) between autistic and control lymphoblasts (Figure 5 and Table 1).

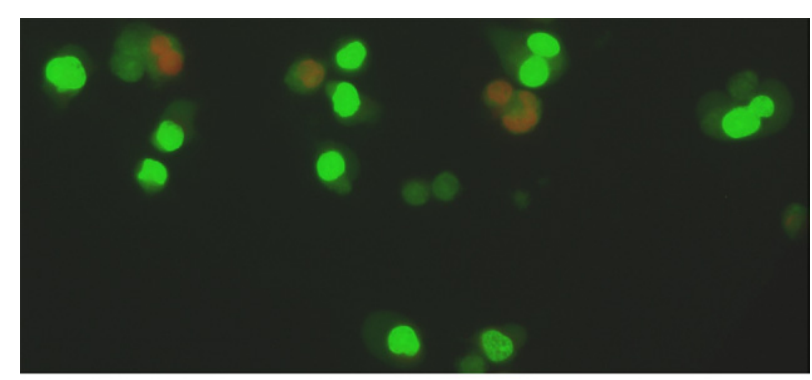

Control

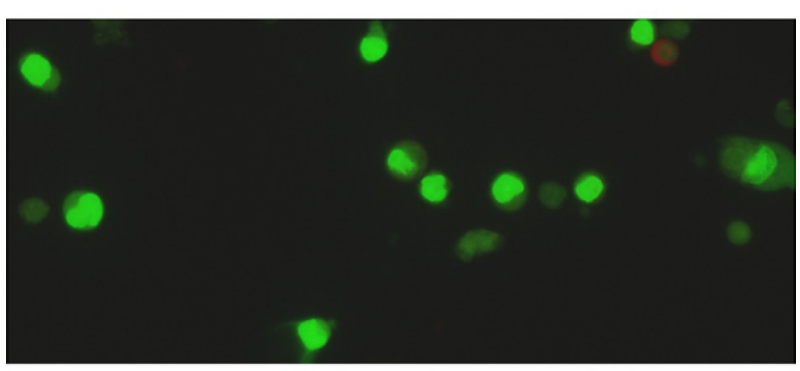

Autism

Figure 5. Fluorescence staining of lymphoblasts: A representative image of lymphoblast cells from six autistic subjects and six controls after acridine orange (AO) plus propidium iodide (PI) double staining. Cells were examined at $\times 400$ magnification with a fluorescence microscope (Nikon). AO stained live cells with intact cytoplasm (green) and nuclei (bright green), and PI stained dead/dying cells. The nuclear and cytoplasmic areas of viable lymphoblast cells were measured by Image-Pro Plus software.

\section{Overexpression of FAK in Autistic Lymphoblasts Rescues Migration and Adhesion Defects}

To further evaluate whether compromised FAK-Src signaling is responsible for the migration and adhesion defects in autistic lymphoblasts, we overexpressed FAK in autistic lymphoblasts and determined the effects. Our results showed that the reduced migration in autistic lymphoblasts was rescued by the overexpression of active His-FAK fusion protein in autistic lymphoblasts (one-way analysis of variance: $F=60.36, P<0.001$; Fisher's PLSD: $P<0.001$; Figure 6A). In addition, we found that the overexpression of FAK could counter the increased adhesion in autistic lymphoblasts (one-way analysis of variance: $F=14.49, P<0.001$; Fisher's PLSD: $P<$ 0.001 ; Figure $6 \mathrm{~B}$ ). These results suggest that FAK plays an important role in the regulation of autistic lymphoblast migration and adhesion.

Table 1. Data for MNA, MCA, and N/C Ratio

\begin{tabular}{crrc}
\hline Variable & Control group & Autism group & $P$ value \\
\hline MNA $\left(\mu \mathrm{m}^{2} \times 10^{3}\right)$ & $30.16 \pm 2.30$ & $28.77 \pm 2.82$ & 0.71 \\
MCA $\left(\mu \mathrm{m}^{2} \times 10^{3}\right)$ & $66.53 \pm 8.00$ & $59.05 \pm 8.66$ & 0.54 \\
N/C ratio & $0.47 \pm 0.03$ & $0.50 \pm 0.03$ & 0.44 \\
\hline
\end{tabular}

Data are given as mean \pm SEM

N/C, nuclear/cytoplasmic; MNA, mean nuclear area; MCA, mean cytoplasmic area. 

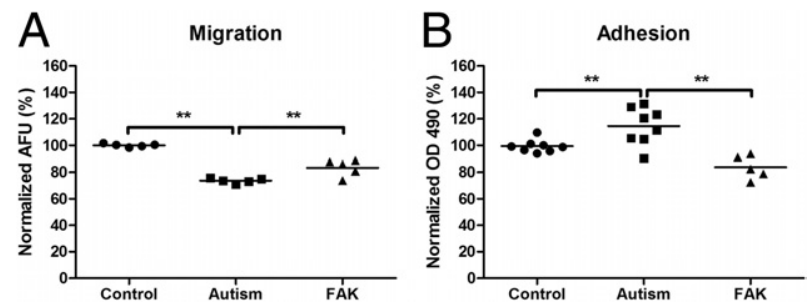

Figure 6. The effect of FAK overexpression on autistic lymphoblast migration and adhesion. A: Migration assays on control lymphoblasts, autistic lymphoblasts, and autistic lymphoblasts treated with FAK. The number of migrated cells was determined by fluorescence reading at 14 hours. The values are mean $\pm \mathrm{SE}$ from five replicates. B: Adhesion assay on control lymphoblasts, autistic lymphoblasts, and autistic lymphoblasts treated with FAK. Adherent cells were quantitated by the colorimetric aqueous MTS assay. The values are mean $\pm \mathrm{SE}$ from five replicates. AFU indicates arbitrary fluorescence units. ${ }^{* *} P<0.01$.

\section{FAK Mediates Its Effect in Autistic Lymphoblast Migration through Src, PI3K-AKt, and MEK Signaling Cascades}

Previous studies ${ }^{24,25}$ have shown that FAK facilitates the generation of integrin-stimulated signals to downstream targets, including Src, MEK signaling cascades, and PI3K-Akt signaling. Thus, we tested whether the stimulation from Src, MEK, PI3K, and Akt was involved in the mechanism of FAK-induced cell migration in autism by treating cells with the selective Src inhibitor (SU6656), PI3K inhibitor (wortmannin), Akt kinase inhibitor, and MEK inhibitor (PD98059). Our results showed that the Src inhibitor completely blocked the stimulating effect of FAK on autistic lymphoblasts and reduced the total migration by $11 \%$ at the given concentration (one-way analysis of variance: $F=8.65, P<0.001$; Fisher's PLSD: $P<0.001$ ). The inhibitors of PI3K and Akt also fully blocked the stimulating effect of FAK on autistic lymphoblasts and reduced the total migration by $12.1 \%$ (Fisher's PLSD: $P<$

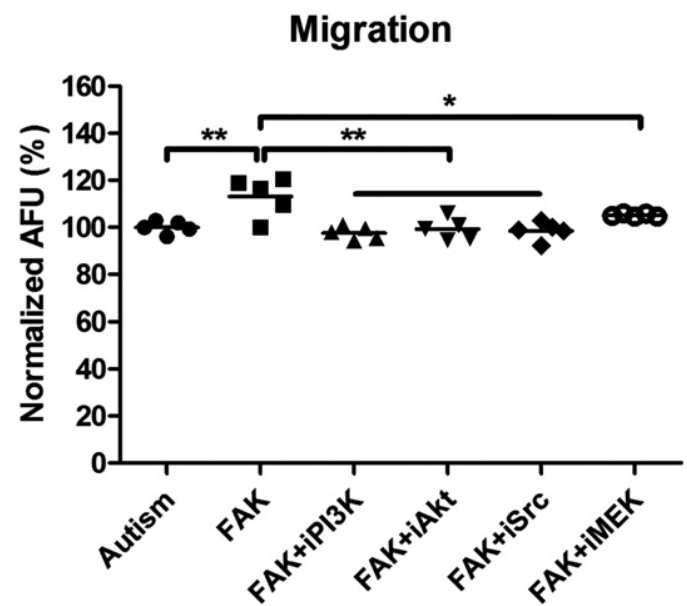

Figure 7. Effect of selective inhibitors on FAK-mediated autistic lymphoblast migration. Migration assays on autistic lymphoblasts treated with FAK, FAK plus Src inhibitor (SU6656), FAK plus PI3K inhibitor (wortmannin), FAK plus Akt kinase inhibitor, or FAK plus MEK inhibitor (PD98059) (i indicates inhibitor). The number of migrated cells was determined by fluorescence reading at 14 hours. The values are mean \pm SE from five replicates. AFU indicates arbitrary fluorescence units. ${ }^{*} P<0.05,{ }^{* * *} P<0.01$
A

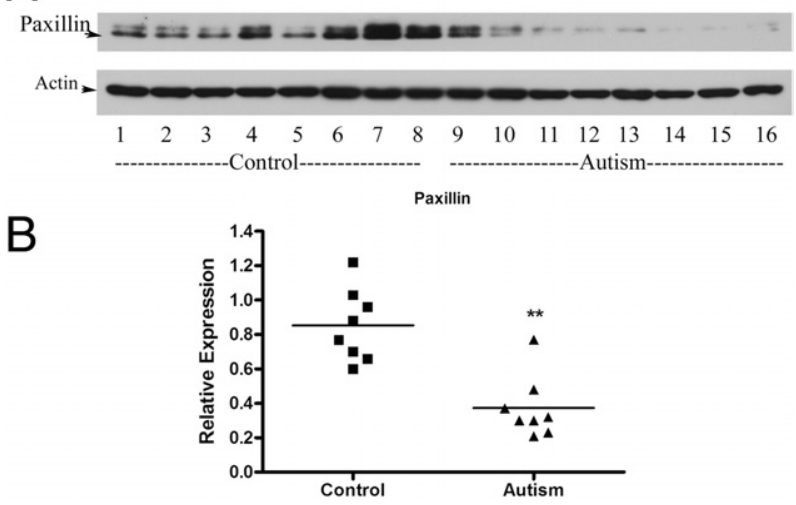

Figure 8. Paxillin protein expression in autistic lymphoblasts. A: Western blot analyses of lymphoblast lysates using paxillin antibody. B: The blot shown in $\mathbf{A}$ was quantitated after normalization by actin. Data are shown as mean $\pm \mathrm{SE}(n=8)$. ${ }^{* k} P<0.01$.

0.001 ) and $10.7 \%$ (Fisher's PLSD: $P<0.001$ ), respectively. The MEK inhibitor blocked the effect of FAK by $85 \%$ and reduced the total migration by $7.7 \%$ (Fisher's PLSD: $P=0.01$; Figure 7 ). These results suggest that FAK exerts its stimulating effect on autistic lymphoblast migration through the interaction with Src and modulation of PI3K-Akt and MEK signaling cascades.

\section{Paxillin Is Likely Involved in the FAK Protein Complex that Regulates Lymphoblast Migration in Autism}

Cell migration is an integrated process that requires the coordinated regulation of various signaling molecules. ${ }^{34,35}$ Previous studies ${ }^{36}$ have shown that the FAKSrc complex can also mediate the phosphorylation of a well-known scaffolding molecule, paxillin, to regulate the organization of the actin cytoskeleton and cell migration. To determine whether paxillin is mediated by the FAK-Src complex to regulate the cell migration in autism, we examined the protein expression of paxillin in autistic lymphoblasts. Our results showed that paxillin was decreased by $56 \%$ in autistic lymphoblasts compared with controls $(t=$ -4.891 , df $=14, P<0.001$; Figure 8 ), suggesting that paxillin is likely involved in the FAK protein complex that regulates lymphoblast migration in autism.

\section{Discussion}

This study provides insight into the mechanisms underlying lymphoblast cell adhesion, migration, and IgG production in autistic subjects. For the first time, to our knowledge, we show that the protein expression levels of both integrin $\beta 1$ and FAK are significantly decreased in autistic lymphoblasts. Unfortunately, we could not detect the phosphorylation levels of FAK using Western blot analysis and believe they are too low for detection. However, we detected that the Src protein expression and the phosphorylation of the active site (Y416) are also significantly reduced in autistic lymphoblasts. These results 
suggest down-regulated integrin $\beta 1$ and FAK-Src signaling activity in autistic lymphoblasts. Previously, several studies have used the lymphoblast cell lines from AGRE to determine the alterations of certain gene expression in autism. One of them reported reduced integrin $\beta 1$ expression in autistic samples using a microarray approach, which supports our findings. ${ }^{37-41}$ FAK and Src family members are the major proteins localized to FAs, which are sites of cell attachment to the ECM. Reelin has exerted a proteolytic activity on ECM proteins through the activation of integrin $\beta 1$. It is possible that the impaired FAK-Src activities may result from reduced levels of reelin and integrin $\beta 1$ in autistic subjects. ${ }^{14,15}$

FAK-Src signaling has activated several signaling pathways through phosphorylation and protein-protein interactions to promote cell survival, cell cycle progression, cell adhesion, migration, and proliferation. ${ }^{20}$ Thus, we further determined whether the down-regulation of FAK-Src signaling affects autistic lymphoblasts adhesion, migration, and proliferation. Although the proliferation of autistic lymphoblasts showed no significant difference compared with controls, the migration and adhesion properties of autistic lymphoblasts were significantly altered compared with those of controls. In addition, we also found that the production of $\operatorname{lgG}$, which is the natural function of $B$ lymphocytes, is impaired in autism. Recently, Heuer et al ${ }^{42}$ reported reduced levels of Igs, including IgG and IgM, in the plasma of autistic children. The reduction in specific Ig levels correlated with behavioral severity (ie, those patients with the highest scores in the behavioral battery had the most reduced levels of IgG and IgM, suggesting an underlying defect in immune function in autism). Our findings of decreased IgG production in cultured autistic $B$ lymphoblasts support this notion. Whether the impairment in IgG production is attributed to the altered adhesion and migration properties in autistic lymphoblasts requires further investigation.

Our results further demonstrated that the adhesion and migration defects in autistic lymphoblasts could be rescued by overexpression of FAK. These findings suggest that FAK-Src signaling plays a crucial role in the regulation of cell adhesion and migration. The down-regulation of the FAK-Src pathway may be the underlying cellularmolecular mechanism responsible for the abnormally altered adhesion and migration in autistic lymphoblasts. Previously, Kanakry et al ${ }^{43}$ demonstrated impairment in neuroligin 1-mediated migration in lymphoblasts derived from patients with schizophrenia. Whether the adhesion and migration defects in autistic lymphoblasts reflect an impairment in the immune system, which may be associated with the pathogenesis of autism, is of significance for further investigation. Several studies have revealed important functional roles of FAK in cortical basement membrane assembly, neural migration, dendritic morphological characteristics, axonal branching, and synapse formation. ${ }^{27-31}$ Thus, B lymphocytes could be an applicable cell model for evaluations of neurodevelopmental disorders. Our next study will investigate whether FAK and its signaling pathways are abnormally regulated in the autistic brain and whether abnormal FAK signaling leads to defects in neural migration, dendritic morpho- logical characteristics, and synapse formation, which could be associated with the pathogenesis of autism.

There is a significant difference $(P=0.046)$ between the mean ages of the eight autistic subjects and the eight control siblings based on the available information. We considered the age factor in the study and analyzed whether age is correlated with the expression of proteins we examined. The ages of autistic subjects vary from 5 to 10 years; however, we found that the protein expression of integrin, FAK, and Src is not correlative with age, suggesting that age has no significant effect on our results. The same analyses have been performed in cell adhesion and migration studies, and no correlation was found between the age and the values of cell adhesion and migration. In addition, several studies ${ }^{44-46}$ from other groups reported that there is no effect of age on the expression of integrin and FAK. In the study subjects, three of the autistic children have a medication history of taking Prozac and carbamazepine. By searching the literature, we did not find any study that documented the effect of Prozac and carbamazepine on B lymphoblasts. We analyzed the data from the subjects with or without the medicine treatment and found no significant difference between the treated and untreated groups.

Our work further elucidated how FAK interacts with its downstream signaling pathways to regulate lymphoblast migration in autism. We showed that FAK mediates its effect through Src, PI3K-Akt signaling, and MEK signaling cascades in autistic lymphoblasts. In addition, we demonstrated that paxillin is likely involved in the FAK protein complex that regulates lymphoblast migration in autism. The activated FAK-Src complex may also protect cells from anoikis, a form of apoptosis induced by cell detachment from the ECM. ${ }^{47,48}$ Previously, we found that the apoptosis-related proteins $\mathrm{Bcl}-2$, p53, and cathepsin $\mathrm{D}$ are altered in both the brain and the lymphoblasts of autistic subjects. ${ }^{4,32,49}$ The pathogenesis of autism may involve enhanced apoptosis in the brain and lymphoblasts. It is possible that the down-regulation of FAK-Src activities may contribute to an alteration of apoptosisrelated proteins in autism.

The PI3K-Akt pathway plays an important role in promoting cell survival and inhibiting cell apoptosis. ${ }^{50,51}$ Recently, we showed that the brain-derived neurotrophic factor-Akt-Bcl-2 pathway was down-regulated in autistic brain specimens. ${ }^{49}$ We reason that the down-regulation of Akt-Bcl-2 could partially result from compromised FAKSrc complex signaling in autism. The PI3K-Akt and MEKERK pathways interact with each other to regulate cell growth and apoptosis. ${ }^{52}$ Both of these pathways are commonly thought to have antiapoptotic and drug resistance effects on cells. ${ }^{52}$ In addition, the MEK-ERK and PI3K-Akt pathways have also interacted with the p53 pathway. Some of these interactions can result in controlling the activity and subcellular localization of apoptoticrelated proteins, such as Bak, Bax, and Bcl-2. ${ }^{53}$ Thus, the down-regulation of FAK signaling could result in alterations in PI3K-Akt-Bcl-2 and MEK-ERK signaling, and the p53 pathway, which could significantly contribute to the pathogenic change in cell growth and apoptosis possibly associated with autism. 
In summary, our studies showed that the autistic Blymphoblast properties of cell migration and adhesion were altered. The Ig formation and secretion capacities of autistic B lymphoblasts were impaired. In addition, our findings suggest that compromised integrin-FAK-Src signaling and reduced paxillin activities could be responsible for the observed abnormalities of $B$ lymphoblasts in autism because overexpression of FAK could rescue the adhesion and migration defects. FAK mediates its effect in lymphoblast migration through the activation of Src, PI3K-Akt, and MEK signaling cascades. These various abnormalities could be used to develop an approach for an early autism diagnosis.

\section{Acknowledgments}

We thank the AGRE Consortium for providing resources and the AGRE families for their participation. The AGRE Consortium includes Dan Geschwind, M.D., Ph.D. (UCLA, Los Angeles, CA); Maja Bucan, Ph.D. (University of Pennsylvania, Philadelphia, PA); W. Ted Brown, M.D., Ph.D., F.A.C.M.G. (N.Y.S. Institute for Basic Research in Developmental Disabilities, Staten Island, NY); Rita M. Cantor, Ph.D. (UCLA School of Medicine, Los Angeles, CA); John N. Constantino, M.D. (Washington University School of Medicine, St. Louis, MO); T. Conrad Gilliam, Ph.D. (University of Chicago, Chicago, IL); Martha Herbert, M.D., Ph.D. (Harvard Medical School, Boston, MA); Clara Lajonchere, Ph.D. (Cure Autism Now, Los Angeles, CA); David H. Ledbetter, Ph.D. (Emory University, Atlanta, GA); Christa Lese-Martin, Ph.D. (Emory University, Atlanta, GA); Janet Miller, J.D., Ph.D. (Cure Autism Now, Los Angeles, CA); Stanley F. Nelson, M.D. (UCLA School of Medicine, Los Angeles, CA); Gerard D. Schellenberg, Ph.D. (University of Washington, Seattle, WA); Carol A. Samango-Sprouse, Ed.D. (George Washington University, Washington, DC); Sarah Spence, M.D., Ph.D. (UCLA, Los Angeles, CA); Matthew State, M.D., Ph.D. (Yale University, New Haven, CT); and Rudolph E. Tanzi, Ph.D. (Massachusetts General Hospital, Boston, MA).

\section{References}

1. Abrahams BS, Geschwind DH: Advances in autism genetics: on the threshold of a new neurobiology. Nat Rev Genet 2008, 9:341-355

2. Li X, Chauhan A, Sheikh AM, Patil S, Chauhan V, Li XM, Ji L, Brown T, Malik M: Elevated immune response in the brain of autistic patients. J Neuroimmunol 2009, 207:111-116

3. Molloy CA, Morrow AL, Meinzen-Derr J, Schleifer K, Dienger K, Manning-Courtney P, Altaye M, Wills-Karp M: Elevated cytokine levels in children with autism spectrum disorder. J Neuroimmunol 2006, 172: 198-205

4. Sheikh AM, Li X, Wen G, Tauqeer Z, Brown WT, Malik M: Cathepsin D and apoptosis related proteins are elevated in the brain of autistic subjects. Neuroscience 2010, 165:363-370

5. Vargas DL, Nascimbene C, Krishnan C, Zimmerman AW, Pardo CA: Neuroglial activation and neuroinflammation in the brain of patients with autism. Ann Neurol 2005, 57:67-81

6. Weiss LA: Autism genetics: emerging data from genome-wide copynumber and single nucleotide polymorphism scans. Expert Rev Mol Diagn 2009, 9:795-803
7. Buxbaum JD, Baron-Cohen S, Devlin B: Genetics in psychiatry: common variant association studies. Mol Autism 2010, 1:6

8. Devlin B, Melhem N, Roeder K: Do common variants play a role in risk for autism? evidence and theoretical musings. Brain Res 2011, 1380: 78-84

9. Pinto D, Pagnamenta AT, Klei L, Anney R, Merico D, Regan R, et al. Functional impact of global rare copy number variation in autism spectrum disorders. Nature 2010, 466:368-372

10. Weiss LA, Arking DE; Gene Discovery Project of Johns Hopkins the Autism Consortium: A genome-wide linkage and association scan reveals novel loci for autism. Nature 2009, 461:802-808

11. Persico AM, Bourgeron T: Searching for ways out of the autism maze: genetic, epigenetic and environmental clues. Trends Neurosci 2006, 29:349-358

12. Kubo K, Honda T, Tomita K, Sekine K, Ishii K, Uto A, Kobayashi K, Tabata H, Nakajima K: Ectopic Reelin induces neuronal aggregation with a normal birthdate-dependent "inside-out" alignment in the developing neocortex. J Neurosci 2010, 30:10953-10966

13. Pollard KS, Salama SR, Lambert N, Lambot MA, Coppens S, Pedersen JS, Katzman S, King B, Onodera C, Siepel A, Kern AD, Dehay C, Igel $\mathrm{H}$, Ares M Jr, Vanderhaeghen P, Haussler D: An RNA gene expressed during cortical development evolved rapidly in humans. Nature 2006, 443:167-172

14. Fatemi SH, Stary JM, Egan EA: Reduced blood levels of reelin as a vulnerability factor in pathophysiology of autistic disorder. Cell Mol Neurobiol 2002, 22:139-152

15. Fatemi SH, Snow AV, Stary JM, Araghi-Niknam M, Reutiman TJ, Lee $\mathrm{S}$, Brooks Al, Pearce DA: Reelin signaling is impaired in autism. Biol Psychiatry 2005, 57:777-787

16. Quattrocchi CC, Wannenes F, Persico AM, Ciafré SA, D'Arcangelo G, Farace MG, Keller F: Reelin is a serine protease of the extracellular matrix. J Biol Chem 2002, 277:303-309

17. Betancur C, Sakurai T, Buxbaum JD: The emerging role of synaptic cell-adhesion pathways in the pathogenesis of autism spectrum disorders. Trends Neurosci 2009, 32:402-412

18. Taniyama Y, Weber DS, Rocic P, Hilenski L, Akers ML, Park J, Hemmings BA, Alexander RW, Griendling KK: Pyk2- and Src-dependent tyrosine phosphorylation of PDK1 regulates focal adhesions. Mol Cell Biol 2003, 23:8019-8029

19. Fraley SI, Feng Y, Krishnamurthy R, Kim DH, Celedon A, Longmore GD, Wirtz D: A distinctive role for focal adhesion proteins in threedimensional cell motility. Nat Cell Biol 2010, 12:598-604

20. Zhao J, Guan JL: Signal transduction by focal adhesion kinase in cancer. Cancer Metastasis Rev 2009, 28:35-49

21. Van Slambrouck S, Jenkins AR, Romero AE, Steelant WF: Reorganization of the integrin alpha2 subunit controls cell adhesion and cancer cell invasion in prostate cancer. Int J Oncol 2009, 34:1717-1726

22. Van Slambrouck S, Grijelmo C, De Wever O, Bruyneel E, Emami S, Gespach C, Steelant WF: Activation of the FAK-src molecular scaffolds and p130Cas-JNK signaling cascades by alpha1-integrins during colon cancer cell invasion. Int J Oncol 2007, 31:1501-1508

23. Crosara-Alberto DP, Inoue RY, Costa CR: FAK signalling mediates NF-kappaB activation by mechanical stress in cardiac myocytes. Clin Chim Acta 2009, 403:81-86

24. Zhu H, Liu XW, Cai TY, Cao J, Tu CX, Lu W, He QJ, Yang B: Celastrol acts as a potent antimetastatic agent targeting beta1 integrin and inhibiting cell-extracellular matrix adhesion, in part via the p38 mitogen-activated protein kinase pathway. J Pharmacol Exp Ther 2010, 334:489-499

25. Lee SH, Lee YJ, Han HJ: Role of hypoxia-induced fibronectin-integrin beta1 expression in embryonic stem cell proliferation and migration: involvement of PI3K/Akt and FAK. J Cell Physiol 2011, 226:484-493

26. van Nimwegen MJ, van de Water B: Focal adhesion kinase: a potential target in cancer therapy. Biochem Pharmacol 2007, 73:597-609

27. Beggs HE, Schahin-Reed D, Zang K, Goebbels S, Nave KA, Gorski J, Jones KR, Sretavan D, Reichardt LF: FAK deficiency in cells contributing to the basal lamina results in cortical abnormalities resembling congenital muscular dystrophies. Neuron 2003, 40:501-514

28. Rico B, Beggs HE, Schahin-Reed D, Kimes N, Schmidt A, Reichardt LF: Control of axonal branching and synapse formation by focal adhesion kinase. Nat Neurosci 2004, 7:1059-1069

29. Watanabe F, Miyazaki T, Takeuchi T, Fukaya M, Nomura T, Noguchi S, Mori H, Sakimura K, Watanabe M, Mishina M: Effects of FAK 
ablation on cerebellar foliation: Bergmann glia positioning and climbing fiber territory on Purkinje cells. Eur J Neurosci 2008, $27: 836-854$

30. Xie Z, Sanada K, Samuels BA, Shih H, Tsai LH: Serine 732 phosphorylation of FAK by Cdk5 is important for microtubule organization, nuclear movement, and neuronal migration. Cell 2003, 114:469-482

31. Baron CA, Tepper CG, Liu SY, Davis RR, Wang NJ, Schanen NC, Gregg JP: Genomic and functional profiling of duplicated chromosome 15 cell lines reveal regulatory alterations in UBE3A-associated ubiquitin-proteasome pathway processes. Hum Mol Genet 2006, 15: 853-869

32. Malik M, Sheikh AM, Wen G, Spivack W, Brown WT, Li X: Expression of inflammatory cytokines: Bcl2 and cathepsin D are altered in lymphoblasts of autistic subjects. Immunobiology 2011, 216:80-85

33. Bjorge JD, Jakymiw A, Fujita DJ: Selected glimpses into the activation and function of Src kinase. Oncogene 2000, 19:5620-5635

34. Becchetti A, Arcangeli A: Integrins and ion channels in cell migration: implications for neuronal development, wound healing and metastatic spread. Adv Exp Med Biol 2010, 674:107-123

35. Webb DJ, Parsons JT, Horwitz AF: Adhesion assembly, disassembly and turnover in migrating cells: over and over and over again. Nat Cell Biol 2002, 4:E97-E100

36. Yun SP, Ryu JM, Han HJ: Involvement of beta1-integrin via PIP complex and FAK/paxillin in dexamethasone-induced human mesenchymal stem cell migration. J Cell Physiol 2011, 226:683-692

37. Baron CA, Liu SY, Hicks C, Gregg JP: Utilization of lymphoblastoid cell lines as a system for the molecular modeling of autism. J Autism Dev Disord 2006, 36:973-982

38. Hu VW, Nguyen A, Kim KS, Steinberg ME, Sarachana T, Scully MA, Soldin SJ, Luu T, Lee NH: Gene expression profiling of lymphoblasts from autistic and nonaffected sib pairs: altered pathways in neuronal development and steroid biosynthesis. PLoS One 2009, 4:e5775

39. Hu VW, Frank BC, Heine S, Lee NH, Quackenbush J: Gene expression profiling of lymphoblastoid cell lines from monozygotic twins discordant in severity of autism reveals differential regulation of neurologically relevant genes. BMC Genomics 2006, 7:118

40. Nishimura Y, Martin CL, Vazquez-Lopez A, Spence SJ, AlvarezRetuerto Al, Sigman M, Steindler C, Pellegrini S, Schanen NC, Warren ST, Geschwind DH: Genome-wide expression profiling of lymphoblastoid cell lines distinguishes different forms of autism and reveals shared pathways. Hum Mol Genet 2007, 16:1682-1698

41. Hu VW, Sarachana T, Kim KS, Nguyen A, Kulkarni S, Steinberg ME, Luu T, Lai Y, Lee NH: Gene expression profiling differentiates autism case-controls and phenotypic variants of autism spectrum disorders: evidence for circadian rhythm dysfunction in severe autism. Autism Res 2009, 2:78-97
42. Heuer L, Ashwood P, Schauer J, Goines P, Krakowiak P, HertzPicciotto I, Hansen R, Croen LA, Pessah IN, Van de Water J: Reduced levels of immunoglobulin in children with autism correlates with behavioral symptoms. Autism Res 2008, 1:275-283

43. Kanakry CG, Li Z, Nakai Y, Sei Y, Weinberger DR: Neuregulin-1 regulates cell adhesion via an ErbB2/phosphoinositide-3 kinase/Aktdependent pathway: potential implications for schizophrenia and cancer. PLoS One 2007, 2:e1369

44. Theocharis SE, Kouraklis GP, Kakisis JD, Kanelli HG, Apostolakou FE, Karatzas GM, Koutselinis AS: Focal adhesion kinase expression is not a prognostic predictor in colon adenocarcinoma patients. Eur J Surg Oncol 2003, 29:571-574

45. Crooks CV, Cross ML, Wall CR: Age-related differences in integrin expression in peripheral blood lymphocytes. Immun Ageing 2010, 7:5

46. Wang F, Shu K, Lei T, Xue D: The expression of integrinbeta1 and FAK in pituitary adenomas and their correlation with invasiveness. $J$ Huazhong Univ Sci Technolog Med Sci 2008, 28:572-575

47. Bouchard V, Demers MJ, Thibodeau S, Laquerre V, Fujita N, Tsuruo T, Beaulieu JF, Gauthier R, Vézina A, Villeneuve L, Vachon PH: Fak/Src signaling in human intestinal epithelial cell survival and anoikis: differentiation state-specific uncoupling with the PI3-K/Akt-1 and MEK/Erk pathways. J Cell Physiol 2007, 212:717-728

48. Bouchard V, Harnois C, Demers MJ, Thibodeau S, Laquerre V, Gauthier R, Vézina A, Noël D, Fujita N, Tsuruo T, Arguin M, Vachon PH: $\beta 1$ integrin/Fak/Src signaling in intestinal epithelial crypt cell survival: integration of complex regulatory mechanisms. Apoptosis 2008, 13: 531-542

49. Sheikh AM, Malik M, Wen G, Chauhan A, Chauhan V, Gong CX, Liu F, Brown WT, Li X: BDNF/Akt/Bcl2 signaling pathway is compromised in the brain of autistic subject. J Neurosci Res 2010, 88:2641-2647

50. Hennessy BT, Smith DL, Ram PT, Lu Y, Mills GB: Exploiting the PI3K/AKT pathway for cancer drug discovery. Nat Rev Drug Discov 2005, 4:988-1004

51. Luo J, Manning BD, Cantley LC: Targeting the PI3K-Akt pathway in human cancer: rationale and promise. Cancer Cell 2003, 4:257-262

52. McCubrey JA, Steelman LS, Chappell WH, Abrams SL, Wong EW, Chang F, Lehmann B, Terrian DM, Milella M, Tafuri A, Stivala F, Libra M, Basecke J, Evangelisti C, Martelli AM, Franklin RA: Roles of the Raf/MEK/ERK pathway in cell growth, malignant transformation and drug resistance. Biochim Biophys Acta 2007, 1773:1263-1284

53. Steelman LS, Pohnert SC, Shelton JG, Franklin RA, Bertrand FE, McCubrey JA: JAK/STAT: Raf/MEK/ERK, PI3K/Akt and BCR-ABL in cell cycle progression and leukemogenesis. Leukemia 2004, 18: $189-218$ 